\title{
EL DERECHO ROMANO Y EL CÓDIGO CIVIL EN EL JAPÓN MODERNO. SISTEMÁTICA, PROPIEDAD Y COPROPIEDAD*
}

\author{
ROMAN LAW STUDIES AND THE CIVIL CODE IN MODERN JAPAN. \\ SYSTEM, OWNERSHIP AND CO-OWNERSHIP
}

\section{TOMOYOSHI HAYASHI*}

\begin{abstract}
RESUMEN: El presente artículo trata de la recepción del Derecho romano en Japón como parte de la modernización de la Era Meiji. Asimismo, desarrolla la sistemática del Código Civil japonés, especialmente en materia de propiedad, para centrarse finalmente en la copropiedad.
\end{abstract}

Palabras clave: Código Civil japonés; Era Meiji; comunidad; propiedad; pandectística.

ABSTRACT: This article refers to the entry of Roman Law into Japan as part of the process of modernization of the Meiji Age. As well, it explains the basic rules of the Japanese Civil Code, specially regarding to ownership, to finally focus in co-ownership.

Key words: Japanese Civil Code; Meiji Age; co-ownership; ownership; pandectística.

\section{INTRODUCCIÓN. LA RECEPCIÓN DEL SISTEMA JURÍDICO OCCIDENTAL Y EL ORIGEN DEL ESTUDIO DEL DERECHO ROMANO EN JAPÓN}

Como historiador japonés del Derecho romano, mi objetivo es mostrar brevemente la experiencia japonesa en el establecimiento de los estudios de Derecho romano como paso a la modernidad, y el establecimiento de un diálogo entre este y el Código Civil japonés. Otros romanistas, con quienes estamos en deuda, en el medio jurídico italiano ya han realizado esfuerzos para explicar la situación del Derecho romano, el Derecho occidental y el Derecho tradicional japonés ${ }^{1}$.

\footnotetext{
* Traducción de Carlos Felipe Amunátegui Perelló, profesor de Derecho romano de la Pontificia Universidad Católica de Chile desde el original titulado Roman Law Studies and the Civil Code un Modern JapanSystem, Ownership, and Co-ownership, publicado en Osaka University Review 55 (2008), pp.15-26 (C) Osaka University Law Review.

${ }^{* *}$ Doctor en Jurisprudencia Universidad de Kyoto, Profesor de Derecho Romano en la Escuela de Graduados de Derecho y Ciencias Políticas de la Universidad de Osaka, Japón, correo electrónico: hayashi_t@ebony.plala.or.jp.

${ }^{1}$ Vg. Muto (1935) p. 299; KamiYa (1992) p. 365; SChiapini (1992) p. 363; Crifò-FusCo (1992) p. 391.
} 
Primeramente, y entrando al concepto de Derechos reales, expondré la situación de los mismos en el Código Civil japonés. A continuación, trataré algunos temas relativos a la propiedad, incluyendo la copropiedad, en cuanto Derecho real.

Japón introdujo un sistema jurídico occidentalizado a fines del siglo XIX, cuando el Derecho civil y el Derecho procesal civil ya se habían separado y su codificación se había completado en las potencias europeas más importantes. Esta particularidad temporal imprimirá su carácter peculiar a los estudios de Derecho romano en Japón.

El sistema jurídico occidental en Japón se formó a partir de códigos, de una manera similar a los modelos europeos en que se basaba, como también en la formación de expertos, tanto académicos como profesionales, que estaban encargados de interpretar los códigos.

Bajo estas circunstancias, no hubo lugar para un usus modernus pandectarum que permitiese a los romanistas participar directamente en la interpretación del Derecho positivo. En todo caso, el estudio del Derecho romano fue introducido como fundamento histórico que subyace los Sistemas Jurídicos Occidentales y como un prerrequisito indispensable para comprenderlo.

Cuando las clases de Derecho occidental comenzaron en 1874 en Tokio KaiseiGakko (que es la precursora de la Universidad de Tokio), la asignatura Derecho romano se enseñaba ya por un profesor inglés, William E. Grigsby ${ }^{2}$. Desde entonces, el Derecho romano ha sido enseñado continuamente a nivel universitario en Japón y puede observarse una importante cantidad de investigación al respecto. Ha sido tratada como una materia separada y distinta del Derecho civil moderno, pero el diálogo entre ambas materias ha continuado hasta el hoy.

El material de clases usado durante el primer período fue una traducción del inglés de las Institutiones de Justiniano, y el sistema de las Institutiones, que comprende derecho de las personas (ius personarum), derechos reales (ius rerum) y derecho de las acciones (ius actionum) fue bastante conocido en el medio japonés de la época ${ }^{3}$. El interés por el Derecho romano fue especialmente fuerte antes de la promulgación del Código Civil.

\section{II: SISTEMÁTICA- LA DISPOSICIÓN GENERAL DEL CÓDIGO CIVIL JAPONÉS Y ESPECIALMENTE DE LOS DERECHOS REALES}

Como es bien conocido, un estudioso francés, Gustave Emile Boissonade de Fontarabie ${ }^{4}$ preparó un borrador de Código Civil que consistía en cinco partes profunda-

\footnotetext{
2 YATA (1934) p. 88.

3 El sistema de las Institutiones, es decir, la división del Derecho en personas, cosas y acciones, fue establecido por Gayo en sus Institutas. De ahí pasa a las Institutiones de Justiniano y es recogido, por tanto, en la sistemática del Código Civil francés y, por ende, en nuestro Código. Consiste en la división de la enseñanza del Derecho (y por tanto del Código) en cuatro libros, el primero dedicado a las personas, el segundo a los bienes, el tercero a la herencia y el cuarto a las obligaciones. Para más detalles sobre la sistemática de Gayo, vid GUZMÁn BRITO (2007) pp. 427-458 (n. del t.).

4 STOLLEIS (1995) p. 95.
} 
mente influido por el Código Civil francés, que, a su vez, tomó la sistemática de las Institutiones ${ }^{5}$. En todo caso, luego de su publicación en 1890, su borrador estuvo envuelto en un serio conflicto académico y político conocido como "Hohten-Ronsoh" y se frustraron sus resultados. De ahí en adelante fue conocido como el Kyuh Minpoh (El Viejo Código Civil) ${ }^{6}$.

Después del conflicto, tres académicos japoneses, Kenjiroh Ume, Yatsuka Hozumi y Masaakira Tomii ${ }^{7}$ fueron comisionados para redactar el borrador del nuevo Código Civil, que entró en vigencia en 1898.

Aunque ninguno de los comisionados era romanista en el sentido estricto de la palabra, tanto Ume como Tomii tenían un profundo conocimiento del Derecho romano. Como académicos de Derecho civil ambos habían escrito sus tesis doctorales en la Universidad de Lyon y habían dado gran peso al estudio del Derecho romano ${ }^{8}$.

Este Código Civil (en adelante el Código Civil japonés) ha sido reformado frecuentemente desde entonces, pero manteniendo una fundamental continuidad hasta el presente. Tiene cinco partes $^{9}$, comenzando por una parte general y terminando por la sucesión por causa de muerte ${ }^{10}$.

Una vez asentado lo anterior, trataremos específicamente los derechos reales. Una de las principales características del Código Civil japonés fue la adopción del sistema alemán de las pandectas. Para aquellos que están familiarizados solo el sistema de las Institutas de Gayo o las Institutiones de Justiniano, la forma en que el Código Civil japonés trata los derechos reales es algo extraña. Las personas son consideradas sujetos de derecho y las cosas objetos de derecho y ambas están definidas claramente en la Parte I "Disposiciones Generales". Luego, la Parte II "Derechos Reales" trata sobre los derechos reales en detalle. En todo caso, los modos de adquirir y perder el dominio están tratados en la Parte III "Derecho de las Obligaciones" y en la Parte V "Derecho de herencias". No obstante que el sistema jurídico occidental fue la influencia dominante en la sustancia del Código Civil japonés, este representa una discusión no resuelta entre académicos del Derecho civil e historiadores del Derecho. Su composición fue claramente influenciada por la Pandectística alemana del XIX. Curiosamente, desde entonces la mayor parte de los textos de Derecho romano fueron compuestos según el sistema pandectístico, a pesar de que hasta entonces habían seguido el sistema de las Institutiones. Yoshijiro Okamoto, quien escribió su tesis doctoral en la Universidad de Leipzig, enseñó Derecho romano desde 1899 hasta 1907 en la Meiji Hohritsu Gakkoh, el antecedente de la Universidad de Meiji ${ }^{11}$. En el prefacio de su texto de Derecho romano, el lamenta que se preste mayor atención a las interpretación del Código Civil japonés que

\footnotetext{
${ }^{5}$ MAEdA (2004) pp. 611, 942-945.

${ }^{6}$ MAKI-FUJIWARA (1993) pp. 351-356.

7 STOLLEIS (1995) p. 295, 618 y 627.

${ }^{8}$ HARAdA (1942) p. 296.

${ }^{9}$ Es decir, sigue el sistema pandectístico (n. del T).

${ }^{10}$ MAKI-FUJIWARA (1993) pp. 356-365.

11 YATA (1934) p. 596.
} 
al Derecho romano entre los académicos e insiste en la importancia del Derecho romano como contexto histórico del Código ${ }^{12}$. El estructuró su manual según el sistema pandectístico.

\section{PROPIEDAD - ESENCIA Y LÍMITES DE LA PROPIEDAD EN EL CÓDIGO CIVIL JAPONÉS}

El cambio desde el sistema premoderno de poder sobre las cosas a la noción moderna de propiedad, paralelo al dominio en los países occidentales, tiene lugar durante el desarrollo de Japón en la Era de Meiji que comienza en $1868^{13}$. No obstante, el momento preciso de esta transformación es objeto de debate ${ }^{14}$.

El Código Civil japonés introdujo la protección de la propiedad privada desde que entro en vigencia. Esta regla conforma el principio mayor del Derecho civil japonés, junto con los principios de "libertad contractual" y "responsabilidad por culpa"15.

Art. 206: "El dueño tiene el derecho de usar, gozar y disponer de sus cosas dentro de los límites de la Ley y los reglamentos".

Según el comentario de De Becker a este artículo, "La propiedad (Shoyu-ken) es un derecho en virtud del cual una cosa puede ser gobernada libremente; en otras palabras, es un derecho de usar libremente, para recibir los beneficios que genere la cosa, y para disponer de ella"16. Esta noción de trilogía (usar, gozar y disponer) es, en ocasiones, atribuida al Derecho romano, especialmente por los académicos de Derecho civil en Japón ${ }^{17}$. En todo caso, ya el gran romanista japonés del siglo XX, Harada, hizo notar en 1937 que la máxima legal "Dominium es ius utendi et abutendi re sua, quatenus iuris ratio patitur" (El dominio es el derecho para usar y disponer del una cosa, hasta donde el Derecho lo permita) solo puede reputarse al siglo XVI como muy temprano ${ }^{18}$.

De entre todas las cosas, a los bienes raíces, y sobre todo a la tierra, se le dio una particular importancia en el Derecho civil japonés. En Japón, el gobierno admitió el dominio privado de la tierra con la Chiken-kofu (promulgación de la Ley de Tierras) en 1872, confirmándose la permisión con el Código Civil. El artículo 207 prescribe:

"La propiedad de la tierra tiene efectos por sobre y debajo de la tierra, dentro de los límites legales y reglamentarios”.

\footnotetext{
12 OKамото (s/a) p. 2.

${ }^{13}$ La Era de Meiji (1867-1912) corresponde al período de modernización de Japón, que abandona su Edad Feudal particular (encarnada en el shogunato Tokugawa) para iniciar un período de rápidas transformaciones sociales y culturales que la llevarán a convertirse en el país más desarrollado y occidentalizado de Asia. En lo jurídico, este período marca la occidentalización de su Derecho y la adopción de un modelo romano germánico (n. del T).

${ }^{14}$ KAI et al. (1979) pp. 167-185.

15 ENDO et al. (2002) PP. 10-14.

16 DE BECKER (1921) p. 151

${ }^{17} \mathrm{Vg}$. ENDO et al. (2003) p. 168.

${ }^{18}$ HARADA (1954) p. 104.
} 
Naturalmente, los dueños de tierras japoneses no pueden reclamar derechos a los brasileños que están en las antípodas, y, además de los límites naturales y razonables del derecho, hay leyes especiales que restringen los efectos del derecho, como la Dai-shindo Chika no Kohkyoh-teki Riyoh ni kansuru Tokubetsu Sochihoh (Ley acerca de las Medidas Especiales que Conciernen el uso público del Subsuelo Profundo), promulgada en 2000.

Este artículo ha sido criticado ya desde 1924 en Japón por el profesor Yoshitaro Hirano, tomando como base la argumentación que en el XIX Otto von Gierke hiciese al primer borrador del BGB, cuyo correspondiente es el artículo 207 del Código Civil Japonés ${ }^{19}$. Dicho argumento será tratado detalladamente en el próximo acápite.

Nuevamente, en las obras recientes de Derecho civil se imputa al Derecho romano la concepción de un dominio que va desde el cielo hasta las profundidades del globo terráqueo. Harada refuta esta crítica tradicional e insiste en que esta noción usque ad caelum (desde el cielo) y usque ad inferos (hasta el profundo subsuelo) viene de los glosadores (específicamente de una nota a Paulus as edictum 21 lib. D. 8,2,1 pr) ${ }^{20}$ y no es atribuible al Derecho romano clásico ${ }^{21}$. Este argumento lo tomó de Pampaloni ${ }^{22}$.

Puesto que mi trabajo está enfocado en el análisis de la academia y el Derecho en el Japón moderno, la formación histórica de esta doctrina en sí misma desde la ciencia jurídica medieval europea escapa a mis propósitos. Por ello me limitaré simplemente a mostrar que el origen texto romano clásico desde donde se formó esta doctrina tenía efectos limitados y poco que ver con unos efectos usque caelum y usque inferos en la propiedad inmobiliaria.

La fuente original quia caelum, quod supra id solum intercedit, liberum esse debet (D.8,2,1 pr. Paulus ad edictum 21) corresponde a la parte subrayada de la siguiente traducción:

"No impide las servidumbres de senda y de paso de ganado ni tampoco la de levantar la altura de un edificio el hecho de que medie <entre el fundo dominante y el sirviente> suelo público o vía pública; en cambio impide la de apoyo de viga, la de voladizo y la de cobertizo, así como la de estilicidio <o sea, de caída de gotas del tejado> y la de caída de canalones, porque el espacio aéreo debe quedar libre" 23

\footnotetext{
${ }^{19}$ Hirano (1924) pp. 79-84.

${ }^{20}$ Así, la Glosa agregó Cuius est solum eius est usque ad coelum (De quien es el suelo, suyo es hasta el cielo). Vid al respecto Viora (1969) p. 296.

${ }^{21}$ Harada (1949) p. 109 y Harada (1954) p. 296.

22 PAMPALONi (1892) p. 34.

${ }^{23}$ En el original se citaba la traducción de WATSON (1998) I:

If public ground or a public roadway lies between two estates, this does not prevent the existence of a servitude of iter or actus or one giving a rght to insert a beam or to have a roof or other rainwater dripping from the eaves of a house [hereafter, eavesdrip]. The reason is that the air space above such ground must be kept clear" Nosotros hemos usado D’ORs (1968) p. 346 como traducción estandarizada.
} 
El argumento de Harada se basa en el conocimiento histórico de la tradición jurídica occidental y en la comprensión de la investigación jurídica contemporánea europea.

De hecho, el problema concerniente a hasta qué punto podían restringirse los derechos privados fue un punto trascendental para la teoría y práctica del Derecho civil de la primera mitad del siglo XX. Más aún, mediante la influencia de las teorías jurídicas alemanas, la Sozialisierung, la socialización de la propiedad, llegó a ser un verdadero lugar común ${ }^{24}$, lo que se concretizó en la teoría del Abuso del Derecho.

Con la aceptación generalizada de dicha doctrina en el mundo académico, los tribunales de justicia gradualmente la aceptaron. El primer caso en que se recibió la doctrina fue el caso Shingenkoh Hatakakematsu, donde una compañía ferroviaria fue demandada por haber arruinado un pino históricamente importante operando locomotoras de vapor sin prevenir las pesadas emisiones de humo. Estas podrían haber sido eliminadas de una manera sencilla si es que la compañía lo hubiese considerado.

La Corte Suprema (Taishinin) admitió dio lugar a la demanda por compensaciones y argumentó que incluso el ejercicio de un derecho, en este caso, la operación de trenes de propiedad de la compañía, puede constituir un delito civil si hay dolo o negligencia del demandado ${ }^{25}$.

Un segundo caso de gran importancia es el de las Termas de Unazuki, donde el la Corte Suprema denegó al dueño (de aquí en adelante A) el derecho a remover una tubería de agua perteneciente a otra persona (en adelante B). A compró el inmueble mediante compra, una tubería que llevaba agua caliente desde una fuente termal hasta un Spa cruzaba la propiedad de A. La tubería pertenecía B, una compañía ferroviaria que era propietaria, además, de un Spa.

A propuso que B debía comprar la tierra por la que pasaba la tubería a un precio muy superior a aquel de mercado y demandó la remoción de la tubería si es que B se negaba a la compra. Debido a que estaba ubicada en un precipicio, su remoción y reubicación habría sido muy costosa. B no aceptó la propuesta de A, por lo que A procedió a demandar a B para que removiera la tubería, de conformidad a su derecho de dominio. La Corte Suprema desechó la demanda como un caso de abuso del derecho ${ }^{26}$.

Más tarde, la doctrina del abuso del Derecho fue incorporada al Código Civil japonés por una reforma de 1947 al artículo $1^{27}$. El texto quedó como sigue:

"1. Los derechos privados deben estar conformes con el bienestar público.

2. El ejercicio de derechos y el cumplimiento de deberes debe ser realizado de buena fe.

3. El abuso del derecho no está permitido”.

\footnotetext{
${ }^{24} \mathrm{Vg}$. KAI et al. (1979) pp. 196-198.

25 Taishinin Minji-Hanketsuroku 25,356, de 19 de Marzo de 1919.

26 ODA (1999) p. 135; Taishinin Minji-Hanreishu 14,1965 de 5 de octubre de 1935.

27 MAEDA (2004) p. 1310-1313.
} 
Desde mi punto de vista, la tendencia a restringir la propiedad privada continuará profundizándose en el futuro con el aumento de varias limitaciones de orden público que restringen su ejercicio.

En todo caso, no debemos ignorar la esfera negativa de la doctrina del abuso del derecho y de la socialización de la propiedad. Según la argumentación de los académicos de Derecho civil, esta doctrina puede, en ocasiones, funcionar deprimiendo los derechos privados de las personas con poco poder y riqueza y promover los intereses de la parte que ya ha establecido el actual estado de cosas que resulta muy costoso de remover.

Otro punto a considerar es que la doctrina de socialización de la propiedad fue sostenida por las teorías nazis del Derecho civil de las décadas de 1930 y 1940, que insistían en la necesidad de movilizar a la nación y su propiedad ${ }^{28}$.

Ocasionalmente, en los debates de Derecho civil que concernían a la protección de derechos sociales y al bien común, a puntos de vista excesivamente individualistas o actitudes que ponían demasiado énfasis en un derecho de propiedad individual fueron atribuidos nombres romanos. Más aún, los especialistas en Derecho Civil no distinguían entre la ciencia pandectística alemana, el Derecho clásico romano y el Derecho medieval, llamándolas todas Derecho romano. A pesar que el mismo Hirano originalmente menciona la diferencia entre los conceptos de pandectística y el Derecho romano en su contexto histórico, tomado de Von Grieke ${ }^{29}$, pareciera, según creo, que esta distinción se hubiese perdido durante la difusión de la ciencia jurídica.

La principal contribución de Harada fue realizar una sobria corrección a esta confusión de conceptos realizada por no especialistas desde la perspectiva de un historiador del Derecho. También explica que el Derecho romano privado funcionaba en la sociedad romana clásica junto con el Derecho público (ius publicum), el derecho divino (fas), la costumbre (mores), la religión y demás. Luego reprocha a los germanistas japoneses el descontextualizar históricamente las instituciones, señalando y amplificando solo algunas de sus características menores ${ }^{30}$. Infiero que esta tesis de pluralidad normativa presente en su apología es producto de la influencia de Fritz Schulz, cuya teoría el introdujo activamente en Japón ${ }^{31}$.

\section{COPROPIEDAD- SU CARÁCTER ROMANO EN EL CÓDIGO CIVIL JAPONÉS Y SUS CIRCUNSTANCIAS}

Como ya he mencionado, hubo un fuerte debate entre germanistas y romanistas en el Japón de la primera mitad del siglo XX, réplica del debate original de la segunda mitad del siglo XIX que se produjo en Alemania, aunque con connotaciones específicamente japonesas.

\footnotetext{
${ }^{28}$ KAI et al. (1979) p. 198.

${ }^{29}$ HiRANO (1924) p. 18.

30 Harada (1949) p. 105; Harada (1950), P. 59.

$31 \mathrm{Vg}$. Harada (1950) I-II.
} 
Uno de los mejores representantes del germanismo en Japón fue Yoshitaro HiRANO, quien comenzó su carrera académica como profesor de Derecho civil y se desarrolló como un teórico del derecho de orientación marxista y activista político. En su primer libro, titulado Minpoh ni okeru Roma-Shisoh to German-Shisoh (Pensamiento Romano y Germano en el Derecho Civil), desarrolla una dura crítica al carácter pandectístico del Código Civil japonés, basando su argumentación primariamente en Von Gierke y, secundariamente, en Anton Menger. Junto con su crítica a la propiedad, es especialmente duro respecto al tratamiento de la copropiedad en el articulado del Código Civil japonés ${ }^{32}$.

El articulado del Código Civil japonés relativo a copropiedad tiene un reconocido carácter romanístico ${ }^{33}$. Cada uno puede disponer libremente de su cuota (art. 206) y la partición siempre es admisible (art. 256, inciso segundo). Este tipo de copropiedad se llama "Kyohyuh" en japonés. Estos conceptos no se aplican a la propiedad común de los socios (art. 667), donde cada socio no puede disponer libremente de su participación (art. 676 inciso segundo). Los académicos del Derecho civil crearon la noción de "Gohyuh" para explicar este segundo tipo de propiedad y distinguirla del Kyohyuh. HirANO critica la noción de Kyohyuh e introduce la idea de "Gesamthand" desde la ciencia jurídica alemana, la que será denominada "Sohyuh", donde cada codueño no puede disponer de su parte ni exigir la partición de la comunidad. Bajo la Sohyuh, los codueños solamente pueden usar y gozar del bien. Esta noción fue aceptada en los estudios de Derecho civil y tenida como aplicable a los derechos comunes (art. 263, art. 294) y a ciertos derechos reales admitidos por la costumbre, como el derecho de uso exclusivo de agua (suiri-ken) o el derecho de uso de aguas termales (onsen-ken). Kyohyuh, Gohyuh y Sohyuh forman las tres categorías de copropiedad en el Derecho civil japonés.

Tomaré los derechos comunitarios como ejemplo (iriai-ken) ${ }^{34}$. Recientemente, los derechos comunitarios atrajeron la atención de los estudiosos del Derecho civil y de los sociólogos como una noción clave para superar la supuesta contraposición de los sectores público y privado de hoy en día, incluso aunque los derechos comunitarios habían gradualmente perdido la atención de los académicos por el paulatino decaimiento poblacional de las villas y la decadencia de los bosques. Este nuevo auge en el interés se ha denominado el "Renacimiento de las Comunidades" 35 . El Derecho civil japonés regula positivamente los derechos comunitarios, aunque de manera muy breve, abandonando su regulación pormenorizada a las costumbres locales sin especificar más. Solo se regula a través de dos artículos:

Art. 263 "Esta sección (copropiedad), junto con las costumbres locales, es aplicable a los derechos comunitarios que tengan la naturaleza de copropiedad".

Art. 294 "Este capítulo (easement XXX), junto con las costumbres locales, se aplica a los derechos comunitarios que no tengan la naturaleza de copropiedad".

\footnotetext{
32 Hirano (1924) pp.165-189.

33 Nishimura - KNÜTEL (2000) pp. 116-129.

${ }^{34}$ DE BECKER (1921) p.178; ENDO et al. (2003) pp. 269-287.
} 
Se desarrolla de la antigua costumbre de permitir a los habitantes de ciertos distritos rurales entrar a ciertos bosques, cotar leña o pasto, juntar ramas caídas u hojas y así, incluso aunque no les pertenecieran. Antes de la Restauración Meiji de 1867, los bosques eran frecuentemente tenidos como de propiedad de los "Mura" (comunidades locales, villas) y sus miembros podían entrar a los bosques para beneficiarse de ellos, aunque sin poder disponer de ellos o exigir su partición. En la reforma que siguió directamente a la "Chiso-kaisei" (Reforma del impuesto territorial) en 1873, la propiedad de a tierra fue dividida en tierra pública y privada (Tochi-kanmin-yuh-kubun), y la propiedad de los bosques fue retenida por el gobierno, salvo que alguno pudiese probar dominio privado. El derecho de los miembros de las comunidades locales para entrar en los bosques podía extenderse, aunque se volvía menos estable. Luego, incluso los bosques privados fueron incorporados a la propiedad de las villas como los estratos más bajos del gobierno Meiji a través del establecimiento de la "Chohson-sei" (La Fundación de Municipios y Villas) en $1888^{36}$. El derecho de los aldeanos para entrar en los bosques podía abolirse discrecionalmente por el Concejo Municipal. Cuando el Código Civil japonés entró en vigencia en 1898, los antiguos derechos que involucran el uso de los bosques cambiaron dramáticamente.

Es en este contexto que las críticas de Hirano acerca de la noción romana de copropiedad adquieren sentido, puesto que terminaron por justificar el despojo de estos derechos más antiguos y premodernos, puesto que la protección de los derechos comunitarios en el Código Civil japonés fue insuficiente a la hora de su promulgación.

En todo caso, debe recalcarse que no todas sus críticas atribuciones de instituciones al Derecho romano eran científicamente exactas desde la perspectiva del historiador del Derecho.

Recientemente, el académico de Derecho civil Yoshioka analizó críticamente la historia de la teoría jurídica del período Meiji acerca de los derechos comunitarios, específicamente en lo relativo al trabajo de Koru Nakata, un famoso estudioso de historia jurídica comparada e historia jurídica japonesa.

\section{CONCLUSIONES Y PERSPECTIVAS}

Los estudios de Derecho romano tienden a hacerse más teóricos e históricos desde mediados del siglo XX. Se concentran más en el Derecho romano en su propio contexto clásico, que en su aplicación práctica en el Derecho contemporáneo. No obstante, su diálogo con el Derecho civil ha continuado, ofreciendo y el Derecho romano una perspectiva comparativa e histórica. No hubo tampoco nuevos debates entre germanistas y romanistas en Japón. Aquí quisiera mencionar a Satoru Yoshino, Tokuji Sato y Takashiro Taniguchi como los más importantes romanistas japoneses que contribuyeron al estudio de los derechos reales en este período ${ }^{37}$. Recientemente, el diálogo y la comunicación

\footnotetext{
35 SUZUKI et al. (2006) p. 16-18.

36 YOSHIOKA (2006) p. 194.

37 Yoshino (1972); Diósdi (1983); TANiguCHI (1999).
} 
entre el Derecho romano y civil se ha hecho muy frecuente y la investigación histórica acerca de los orígenes del Código Civil japonés ha producido interesantes resultados. El congreso internacional de Fukuoka para conmemorar el centenario del Código Civil japonés fue uno de los más grandes logros en esta área ${ }^{38}$.

\section{BIBLIOGRAFÍA CITADA}

CrIfò, Giuliano y FUSCO, Sandro Angelo (1992): "Sul primo Simposio romanistico internazionale giapponese: Fukuoka 1991”, Index XX pp. 391-403.

De BeCKer, J.E. (1921): The Principles and Practice of the Civil Code in Japan, A Theoretical and Practical Exposition of the motifs of the Japanese Civil Code (Londres, Butterworths) 853 pp.

DiósDI, György (1983): Ownership in Ancient and Preclassical Roman Law (Budapest, 1970) (traducción japonesa de SATO, Tokuji et al. Roma Shoyuhkenhoh no Riron (Tokio, Gakuyohshoboh) 334 pp.

D’Ors, Álvaro et al. (1968): El Digesto de Justiniano (Pamplona, Aranzadi) v. I, 736 pp.

ENDO, Hiroshi et al. (2002): Minpoh I Sohsoku [Derecho Civil y Disposiciones Generales] (Tokio, Yuhikaku) 303 pp.

GUZMÁN BRITO, Alejandro (2006): "El carácter dialéctico del sistema de las Instituciones de Gayo", en: Estudios de Derecho Romano en Homenaje al Prof. Dr. Francisco Samper Polo (Santiago, Librotecnia) 671 pp.

HARADA, Keikichi (1942): Waga Kuni ni okeru Gaikoku Hohshigaku no Hattatsu [Historia del Desarrollo del Derecho Extranjero en Nuestro País] (Tokio) pp. 294-307 en: Tokyo Teikokudaigaku Gakujutsu Taikan, Hohgakubu-Keizaigakubu [A Survey of the Academic Activities at the Tokyo Imperial University, Faculty of Law and Faculty of Economics] (Tokyo, 1942, publication of the Tokyo Imperial University) 786 pp.

HARADA, Keikichi (1949): Roma-hoh [Derecho Romano] (Tokio, Yuhikaku) 408 pp.

HaradA, Keikichi (1950): Roma-hoh no Genri [Los Principios del Derecho Romano] (Tokio, Kohbundoh) 220 pp.

Harada, Keikichi (1954) Nihon Minpohten no Shiteki Sobyoh [Un Trazado Histórico del Código Civil Japonés] (Tokio, Sohbunsha) 396 pp.

Hirano, Yoshitaro (1924): Minpoh ni okeru Roma-shisoh to German-shisoh [El Pensamiento Romano y el Pensamiento Alemán en el Derecho Civil] (Tokio, Yuhikaku) 503 pp.

KAI, Michitaro et al. (1979): Shoyuhken-shisoh no Rekishi [Historia de la Idea de Propiedad] (Tokio, Yuhikaku) 205 pp.

KAMIYA, Norio (1992): Aspetti e problemi della storia giuridica in Giappone: la ricezione del diritto cinese e del sistema romanista, Index XX pp. 365-390.

Nishimura, Shigeo y KNÜTEL, Rolf (2000): Niohn Minpohten to Seiouhoh-dentoh, Nihon Minpohten Hyakunen Kinen Kosusai Symposium [El Código Civil Japonés y la Tradición Jurídica Occidental, Acatas del Simposio Internacional Conmemorativo 
del Centésimo Aniversario del Código Civil Japonés] (Fukuoka, Kyuhshuhdaigakushuppankai) $554 \mathrm{pp}$.

MAedA, Tatsuaki (2004): Siryoh Minpohten [Documentos históricos del Código Civil japonés] (Tokio, Seibundoh) 1919 pp.

MaKi, Hidemasa y FujIWARA, Akihisa (1993): Nihon Hohseishi [Historia del Derecho Japonés] (Tokio, Seirinshoin) 461 pp.

Muto, Toshio (1935): "Il diritto romano e il diritto giapponese" en: Atti del congresso internazionale di diritto romano, Bologna e Roma XVII-XXVII, Aprile MCMXXXIII (Pavia) pp. 299-319.

Oкамото, Yoshijiroh (s/año): Roma-hoh Kohgi [Lecciones de Derecho Romano] (Tokio, Meijidaigaku-shuppanbu) reimpreso (Tokio, 2004, Shinzansha) pp. 381.

ODA, Hiroshi (1999): Japanese Law (Oxford, Butterworths) 444 pp.

PAmpaloni, Muzio (1892): "Sulla condizione giuridica dello spazio aereo e del sottosuolo nel diritto romano e odierno", Archivio Giuridico XLVIII: pp. 32-60.

SCHIAPINI, Sandro (1992): “Giappone e diritto romano: I temi, le prospettive", Index $X X:$ pp. 363-364.

STOLLEIS, Michael (1995): Juristen: ein biographiches Lexikon; von der Antike bis zum 20 Jahrhundert (München, C. H. Beck) 702 pp.

SuZUKI, Tatsuya et al. (2006): Commons-ron Saikoh [Repensando las Comunidades] (Kyoto, Kohyohshoboh) 269 pp.

TANIGUCHI, Takahiro (1999): Roma Shoyuhken Johto-hoh no Kenkyuh [Un Estudio del Derecho de Enajenar la Propiedad] (Tokio, Seibundoh) 267 pp.

Viora, Mario (1969): Acursii Glossa in Digestum Vetus (Torino, Officina Erasmiana) 450 pp.

Watson, Alan (1998): The Digest of Justinian, Revised English-language edition. (Philadelphia, University of Pennsylvania Press, 2 vols.).

YATA, Kazuo (1934): "Meiji jidai no Roma-hoh Kyohiku” [La enseñanza del Derecho romano en la era de Meiji], en: Hohgaku-Shinpoh 44 pp. 409-428, 589-606.

Yoshino, Satoru (1972): Roma Shoyuhken-hoh Shi-ron [Historia del Derecho de Propiedad en Roma] (Tokio, Yuhikaku) 329 pp.

YoshioKa, Yoshimitsu (2006): Hohgaku-teki Iriai-ken Ron no 'Genryuh', Nakata Sohyuh Ron Note [El 'Origen' de la Construcción Legal de los Derechos Comunitarios, Una Nota acerca de la Teoría de Nakata sobre Sohyuh] pp. 191-219 en: Suzuki et al. Commons-ron Saikoh [Repensando las Comunidades] (Kyoto, Kohyohshoboh) pp. 269.

NORMAS CITADAS:

CÓdigo Civil JAPOnÉS:

Art. 1.

Art. 206.

Art. 207.

Art. 256. 


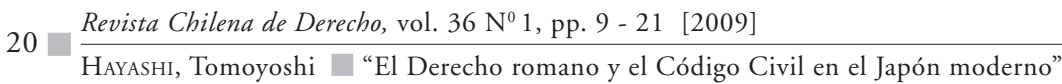

Art. 263.

Art. 294.

Art. 667.

Art. 676.

DigESTO:

D. $8,2,1 \mathrm{pr}$.

\section{SENTENCIAS}

Taishinin Minji-Hanketsuroku 25,356, de 19 de marzo de 1919.

Taishinin Minji-Hanreishu 14,1965 de 5 de octubre de 1935. 